\title{
THE ENHANCEMENT OF PERIPHERAL GLUCOSE UTILIZATION BY GLUCAGON
}

\author{
By H. ELRICK, C. J. HLAD, JR., AND T. WITTEN wITH THE teCHNICAL ASSISTANCE OF \\ T. M. BOW, Y. ARAI, AND A. SMITH \\ (From the Research Laboratories of the Veterans Administration Hospital and the Department \\ of Medicine, University of Colorado School of Medicine, Denver, Colo.)
}

(Submitted for publication June 27, 1955; accepted August 17, 1955)

During the past 30 years evidence has been accumulating which suggests that glucagon (the hyperglycemic-glycogenolytic factor of the pancreas) is a true hormone and that it is produced in the Islets of Langerhans $(1,2)$. Its purification and crystallization were finally accomplished in 1953 by Staub, Sinn, and Behrens (3). The crystalline material is a simple protein with a molecular weight of approximately 4,200 and an amino acid composition which differs in important ways from that of insulin (4). This establishes glucagon as an entity distinct from insulin.

Crystalline glucagon is an extremely active substance. In the cat, $0.05 \gamma$ per $\mathrm{Kg}$. body weight causes a significant rise in blood sugar which lasts 25 minutes (4). Sutherland, Cori, Wosilait, and Rall $(5,6)$ have shown that glucagon stimulates the synthesis of active phosphorylase in the liver. This enzyme catalyzes the rate-limiting reaction (glycogen $\rightleftarrows$ glucose-1-phosphate) in the transformation of glycogen to glucose. The effect of glucagon on liver phosphorylase adequately explains its well-known hyperglycemic-glycogenolytic action.

With respect to its role in carbohydrate metabolism, some investigators look upon glucagon as an insulin antagonist $(1,2,7)$ while others consider it an insulin synergist $(8,9)$. Still others are skeptical of its hormonal nature $(10,11)$. In the present paper experiments are reported which indicate that glucagon, in addition to mobilizing liver glycogen, increases the peripheral utilization of glucose. ${ }^{1}$

\section{METHODS}

Human experiments. Thirty-two male subjects (ages 21 to 47) considered normal with respect to carbohydrate metabolism were studied. All were hospital patients on high caloric and carbohydrate intakes for at least three

1 Glucose utilization is used to mean the disappearance of blood glucose. days before being studied. The test procedure was begun in the morning after a 14-hour fast. Soon after awakening the subjects were placed at bed rest for 30 to 60 minutes in the testing room before the experiment was begun. A 10 per cent solution of glucose was infused intravenously at a constant rate $(250$ to $370 \mathrm{mg}$. per min. in different subjects) by means of a constant infusion pump (Bowman) for periods of 120 to $145 \mathrm{~min}$ utes. In 12 subjects glucagon ${ }^{2}(0.7$ to $1.0 \mathrm{mg}$.) was added to the perfusate following an initial period of 40 to 60 minutes with glucose alone. In 12 subjects the glucose infusion rate was doubled after 50 minutes and after 90 minutes the initial rate was resumed in order to simulate the hyperglycemia caused by glucagon. Two subjects received glucose at a constant rate for 2 hours. The initial control data on 8 other subjects who subsequently received other hormones were also used in the analysis of data.

Glucose determinations (Nelson-Somogyi Method) (12) were done on venous (indwelling needle in antecubital vein) and capillary (finger) blood from the same arm at 5 or 10 -minute intervals, using $0.2-\mathrm{ml}$. samples ( 30 to 40 samples from each subject). Venous samples were drawn without stasis and analyzed in duplicate. (The average difference between duplicate determinations was 2 per cent.) The antecubital vein carries venous drainage from the deep and superficial tissues of the forearm (13-15), while capillary blood has the same glucose content as arterial blood (16-18).

Sodium space was determined in 8 subjects receiving glucagon and 12 subjects receiving glucose alone using radiosodium $\left(\mathrm{Na}^{\mathrm{m}}\right)$ which was added to the infusion fluid. Plasma samples (drawn along with samples for glucose determination) were assayed for radioactivity in a R-C Scientific scintillation well type counter. The method used to calculate sodium space by this technique will be described elsewhere (19).

Dog experiments. The effect of glucagon on arteriovenous glucose differences in the dog was also studied. Six experiments were done in five normal dogs, and nine experiments in three depancreatized dogs. The normal animals were studied under Pentobarbital Sodium anes-

2 Preparations No. 208-158B-214 and No. 208-158B214A kindly supplied by Drs. O. K. Behrens and W. R. Kirtley of the Eli Lilly Company. These preparations have a glucagon potency of 50 per cent of the crystalline standard and an insulin content of between 0.05 to 0.005 unit per mg. 
thesia, after a fast of 16 hours. The depancreatized dogs were tested (under anesthesia) 4 to 16 hours after their last dose of regular insulin and food. Glucagon in doses of 0.072 to $1.5 \mathrm{mg}$. was administered intravenously, either as a single injection or by continuous infusion. Blood samples were drawn without stasis from indwelling needles in the femoral artery and vein. Glucose determinations were done on $1-\mathrm{ml}$. (normal dogs) or $0.5-\mathrm{ml}$. (depancreatized dogs) samples in duplicate.

\section{RESULTS}

\section{Human experiments}

Tables I, II, and III contain the arteriovenous glucose data from the 32 subjects. Two types of response to glucagon were observed. In 11 subjects, blood sugar fell after reaching a peak level, despite the constant infusion of glucagon
(Figure 1-A). In one subject the peak glucose levels persisted throughout the test period. In all subjects glucagon, in addition to producing a sharp rise in blood sugar levels resulted in a marked increase in arteriovenous (A-V) glucose differences. In the 12 subjects in whom the glucose infusion was doubled following the control period, the arterial glucose concentrations closely simulated those observed in the subjects receiving glucagon. However, the $\mathrm{A}-\mathrm{V}$ glucose differences were much less (Figure 1-B). The $A-V / A^{3}$ values of the 12 glucagon periods were compared statistically with those of the 32 control glucose

${ }^{3} \mathrm{~A}-\mathrm{V} / \mathrm{A}=$ arteriovenous glucose difference/arterial glucose concentration. This serves as an index of the peripheral utilization constant of glucose (see appendix for derivation).

TABLE I

Glucagon cases *

\begin{tabular}{|c|c|c|c|c|c|c|c|c|c|c|c|c|c|c|c|c|c|}
\hline \multirow[b]{2}{*}{ Patient } & \multirow[b]{2}{*}{ Age } & & \multicolumn{15}{|c|}{ Time in minutes } \\
\hline & & & 0 & 10 & 20 & 30 & 40 & 50 & 60 & 70 & 80 & 90 & 100 & 110 & 120 & 130 & 140 \\
\hline B. D. & 30 & $\begin{array}{l}\text { At } \\
\text { Vt }\end{array}$ & $\begin{array}{l}86 \\
79\end{array}$ & $\begin{array}{l}98 \\
88\end{array}$ & $\begin{array}{r}107 \\
95\end{array}$ & $\begin{array}{l}112 \\
100\end{array}$ & $\begin{array}{l}115 \\
102\end{array}$ & $\begin{array}{l}117 \\
105\end{array}$ & $\begin{array}{l}118 \\
106\end{array}$ & $\begin{array}{l}137 \\
107\end{array}$ & $\begin{array}{l}172 \\
144\end{array}$ & $\begin{array}{l}187 \\
150\end{array}$ & $\begin{array}{l}178 \\
146\end{array}$ & $\begin{array}{l}162 \\
133\end{array}$ & & & \\
\hline W. D. & 26 & $\stackrel{A}{\mathrm{~V}}$ & $\begin{array}{l}85 \\
85\end{array}$ & $\begin{array}{l}104 \\
101\end{array}$ & $\begin{array}{l}118 \\
111\end{array}$ & $\begin{array}{l}129 \\
118\end{array}$ & $\begin{array}{l}137 \\
124\end{array}$ & $\begin{array}{l}142 \\
127\end{array}$ & $\begin{array}{l}147 \\
129\end{array}$ & $\begin{array}{l}173 \\
152\end{array}$ & $\begin{array}{l}197 \\
167\end{array}$ & $\begin{array}{l}200 \\
174\end{array}$ & $\begin{array}{l}195 \\
164\end{array}$ & $\begin{array}{l}188 \\
130\end{array}$ & & & \\
\hline K. R. & 21 & $\stackrel{\mathrm{A}}{\mathrm{V}}$ & $\begin{array}{l}78 \\
77\end{array}$ & $\begin{array}{r}101 \\
93\end{array}$ & $\begin{array}{l}111 \\
103\end{array}$ & $\begin{array}{l}118 \\
110\end{array}$ & $\begin{array}{l}124 \\
116\end{array}$ & $\begin{array}{l}129 \\
121\end{array}$ & $\begin{array}{l}142 \\
125\end{array}$ & $\begin{array}{l}167 \\
146\end{array}$ & $\begin{array}{l}183 \\
158\end{array}$ & $\begin{array}{l}183 \\
160\end{array}$ & $\begin{array}{l}177 \\
157\end{array}$ & $\begin{array}{l}169 \\
149\end{array}$ & $\begin{array}{l}165 \\
137\end{array}$ & & \\
\hline C. C. & 46 & $\stackrel{\mathrm{A}}{\mathrm{V}}$ & $\begin{array}{l}90 \\
89\end{array}$ & $\begin{array}{l}97 \\
95\end{array}$ & $\begin{array}{l}102 \\
100\end{array}$ & $\begin{array}{l}107 \\
105\end{array}$ & $\begin{array}{l}110 \\
108\end{array}$ & $\mid \begin{array}{l}114 \\
112\end{array}$ & $\begin{array}{l}136 \\
125\end{array}$ & $\begin{array}{l}151 \\
139\end{array}$ & $\begin{array}{l}160 \\
147\end{array}$ & $\begin{array}{l}167 \\
155\end{array}$ & $\begin{array}{l}171 \\
159\end{array}$ & $\begin{array}{l}175 \\
164\end{array}$ & $\begin{array}{l}177 \\
167\end{array}$ & $\begin{array}{l}176 \\
165\end{array}$ & \\
\hline R. L. & 24 & $\begin{array}{l}\mathrm{A} \\
\mathrm{V}\end{array}$ & $\begin{array}{l}80 \\
77\end{array}$ & $\begin{array}{l}89 \\
86\end{array}$ & $\begin{array}{l}95 \\
92\end{array}$ & $\begin{array}{l}99 \\
96\end{array}$ & $\begin{array}{r}101 \\
98\end{array}$ & $\begin{array}{l}150 \\
135\end{array}$ & $\begin{array}{l}184 \\
170\end{array}$ & $\begin{array}{l}207 \\
195\end{array}$ & $\begin{array}{l}222 \\
207\end{array}$ & $\begin{array}{l}230 \\
212\end{array}$ & $\begin{array}{l}211 \\
192\end{array}$ & $\begin{array}{l}193 \\
175\end{array}$ & $\begin{array}{l}177 \\
158\end{array}$ & $\begin{array}{l}164 \\
134\end{array}$ & \\
\hline J. D. & 36 & $\begin{array}{l}\mathbf{A} \\
\mathbf{V}\end{array}$ & $\begin{array}{l}85 \\
82\end{array}$ & $\begin{array}{l}96 \\
94\end{array}$ & $\begin{array}{l}103 \\
100\end{array}$ & $\begin{array}{l}107 \\
102\end{array}$ & $\begin{array}{l}109 \\
103\end{array}$ & $\left\lceil\begin{array}{l}126 \\
111\end{array}\right.$ & $\begin{array}{l}153 \\
139\end{array}$ & $\begin{array}{l}169 \\
157\end{array}$ & $\begin{array}{l}176 \\
158\end{array}$ & $\begin{array}{l}172 \\
150\end{array}$ & $\begin{array}{l}156 \\
142\end{array}$ & $\begin{array}{l}144 \\
133\end{array}$ & $\begin{array}{l}133 \\
122\end{array}$ & $\begin{array}{l}122 \\
113\end{array}$ & $\begin{array}{l}112 \\
106\end{array}$ \\
\hline R. B. & 47 & $\begin{array}{l}\mathbf{A} \\
\mathbf{V}\end{array}$ & $\begin{array}{l}87 \\
82\end{array}$ & $\begin{array}{l}98 \\
92\end{array}$ & $\begin{array}{r}106 \\
98\end{array}$ & $\begin{array}{r}113 \\
94\end{array}$ & $\begin{array}{l}118 \\
106\end{array}$ & $\begin{array}{l}127 \\
108\end{array}$ & $\begin{array}{l}147 \\
125\end{array}$ & $\begin{array}{l}164 \\
139\end{array}$ & $\begin{array}{l}172 \\
141\end{array}$ & $\begin{array}{l}165 \\
135\end{array}$ & $\begin{array}{l}161 \\
120\end{array}$ & $\begin{array}{l}157 \\
118\end{array}$ & $\begin{array}{l}153 \\
116\end{array}$ & $\begin{array}{l}148 \\
114\end{array}$ & $\begin{array}{l}143 \\
114\end{array}$ \\
\hline R. R. & 29 & $\stackrel{\mathbf{A}}{\mathbf{V}}$ & $\begin{array}{l}99 \\
97\end{array}$ & $\begin{array}{l}109 \\
106\end{array}$ & $\begin{array}{l}116 \\
113\end{array}$ & $\begin{array}{l}120 \\
117\end{array}$ & $\begin{array}{l}125 \\
122\end{array}$ & $\mid \begin{array}{l}141 \\
133\end{array}$ & $\begin{array}{l}167 \\
154\end{array}$ & $\begin{array}{l}190 \\
174\end{array}$ & $\begin{array}{l}198 \\
189\end{array}$ & $\begin{array}{l}203 \\
194\end{array}$ & $\begin{array}{l}207 \\
192\end{array}$ & $\begin{array}{l}219 \\
188\end{array}$ & $\begin{array}{l}187 \\
149\end{array}$ & $\begin{array}{l}178 \\
143\end{array}$ & $\begin{array}{l}173 \\
139\end{array}$ \\
\hline F. R. & 34 & $\begin{array}{l}\text { A } \\
\text { V }\end{array}$ & $\begin{array}{l}80 \\
80\end{array}$ & $\begin{array}{l}93 \\
91\end{array}$ & $\begin{array}{l}99 \\
95\end{array}$ & $\begin{array}{r}101 \\
96\end{array}$ & $\begin{array}{r}102 \\
96\end{array}$ & $\mid \begin{array}{l}119 \\
105\end{array}$ & $\begin{array}{l}155 \\
137\end{array}$ & $\begin{array}{l}179 \\
161\end{array}$ & $\begin{array}{l}194 \\
177\end{array}$ & $\begin{array}{l}189 \\
174\end{array}$ & & & & & \\
\hline G. N. & 35 & $\begin{array}{l}\mathbf{A} \\
\mathbf{V}\end{array}$ & $\begin{array}{l}110 \\
105\end{array}$ & $\begin{array}{l}130 \\
123\end{array}$ & $\begin{array}{l}142 \\
136\end{array}$ & $\begin{array}{l}149 \\
144\end{array}$ & $\begin{array}{l}155 \\
150\end{array}$ & $\left\lceil\begin{array}{l}158 \\
153\end{array}\right.$ & $\begin{array}{l}164 \\
157\end{array}$ & $\begin{array}{l}209 \\
194\end{array}$ & $\begin{array}{l}231 \\
214\end{array}$ & $\begin{array}{l}243 \\
215\end{array}$ & $\begin{array}{l}228 \\
196\end{array}$ & $\begin{array}{l}213 \\
189\end{array}$ & $\begin{array}{l}202 \\
183\end{array}$ & $\begin{array}{l}198 \\
182\end{array}$ & \\
\hline R. T. & 30 & $\begin{array}{l}\mathbf{A} \\
\mathbf{V}\end{array}$ & $\begin{array}{l}91 \\
87\end{array}$ & $\begin{array}{l}105 \\
102\end{array}$ & $\begin{array}{l}113 \\
108\end{array}$ & $\begin{array}{l}119 \\
112\end{array}$ & $\begin{array}{l}122 \\
114\end{array} \mid$ & $\left\lceil\begin{array}{l}138 \\
124\end{array}\right.$ & $\begin{array}{l}168 \\
148\end{array}$ & $\begin{array}{l}186 \\
159\end{array}$ & $\begin{array}{l}191 \\
163\end{array}$ & $\begin{array}{l}178 \\
149\end{array}$ & $\begin{array}{l}161 \\
136\end{array}$ & $\begin{array}{l}145 \\
123\end{array}$ & $\begin{array}{l}131 \\
112\end{array}$ & $\begin{array}{l}125 \\
104\end{array}$ & $\begin{array}{r}122 \\
99\end{array}$ \\
\hline G. W. & 32 & $\begin{array}{l}\mathbf{A} \\
\mathbf{V}\end{array}$ & $\begin{array}{l}78 \\
76\end{array}$ & $\begin{array}{l}89 \\
87\end{array}$ & $\begin{array}{l}99 \\
97\end{array}$ & $\begin{array}{l}108 \\
106\end{array}$ & $\begin{array}{l}114 \\
112\end{array}$ & $\left\lceil\begin{array}{l}131 \\
117\end{array}\right.$ & $\begin{array}{l}166 \\
147\end{array}$ & $\begin{array}{l}198 \\
167\end{array}$ & $\begin{array}{l}214 \\
175\end{array}$ & $\begin{array}{l}217 \\
178\end{array}$ & $\begin{array}{l}206 \\
172\end{array}$ & $\begin{array}{l}184 \\
157\end{array}$ & $\begin{array}{l}170 \\
144\end{array}$ & $\begin{array}{l}161 \\
135\end{array}$ & $\begin{array}{l}156 \\
131\end{array}$ \\
\hline
\end{tabular}

* Blood sugar values at 10-minute intervals taken from curves constructed from determinations on samples (30 to 40 per subject) drawn at staggered time intervals which varied in the different subjects.

$\dagger$ A-Capillary blood sugar (mg. per cent), V-Antecubital vein sugar (mg. per cent).

$\uparrow-$ Beginning of glucagon infusion. 
TABLE II

Glucose hyperglycemia cases *

\begin{tabular}{|c|c|c|c|c|c|c|c|c|c|c|c|c|c|c|c|c|}
\hline \multirow[b]{2}{*}{ Patient } & \multirow[b]{2}{*}{ Age } & & \multicolumn{14}{|c|}{ Time in minutes } \\
\hline & & & $\mathbf{0}$ & 10 & 20 & 30 & 40 & 50 & 60 & 70 & 80 & 90 & 100 & 110 & 120 & 130 \\
\hline G. N. & 35 & $\begin{array}{l}\text { At } \\
\text { V }\end{array}$ & $\begin{array}{l}96 \\
92\end{array}$ & $\begin{array}{l}112 \\
104\end{array}$ & $\begin{array}{l}123 \\
114\end{array}$ & $\begin{array}{l}131 \\
122\end{array}$ & $\begin{array}{l}137 \\
128\end{array}$ & $\begin{array}{l}142 \\
133\end{array}$ & $\uparrow \begin{array}{l}166 \\
151\end{array}$ & $\begin{array}{l}183 \\
168\end{array}$ & & $\begin{array}{l}208 \\
194\end{array}$ & $\begin{array}{l}199 \\
196\end{array}$ & $\begin{array}{l}193 \\
190\end{array}$ & $\begin{array}{l}190 \\
187\end{array}$ & $\begin{array}{l}187 \\
185\end{array}$ \\
\hline H. C. & 33 & $\stackrel{\mathrm{A}}{\mathrm{V}}$ & $\begin{array}{l}82 \\
79\end{array}$ & $\begin{array}{l}94 \\
89\end{array}$ & $\begin{array}{r}101 \\
96\end{array}$ & $\begin{array}{l}106 \\
101\end{array}$ & $\begin{array}{l}109 \\
104\end{array}$ & $\begin{array}{l}132 \\
127\end{array}$ & $\begin{array}{l}144 \\
138\end{array}$ & $\begin{array}{l}152 \\
144\end{array}$ & $\begin{array}{l}157 \\
148\end{array}$ & $\begin{array}{l}144 \\
141\end{array}$ & $\begin{array}{l}137 \\
134\end{array}$ & $\begin{array}{l}131 \\
128\end{array}$ & & \\
\hline R. W. & 27 & $\stackrel{\mathrm{A}}{\mathrm{V}}$ & $\begin{array}{l}84 \\
79\end{array}$ & $\begin{array}{l}96 \\
92\end{array}$ & $\begin{array}{l}104 \\
101\end{array}$ & $\begin{array}{l}109 \\
107\end{array}$ & $\begin{array}{l}115 \\
113\end{array}$ & $\begin{array}{l}126 \\
114\end{array}$ & $\begin{array}{l}142 \\
128\end{array}$ & $\begin{array}{l}151 \\
137\end{array}$ & $\begin{array}{l}157 \\
144\end{array}$ & $\begin{array}{l}160 \\
148\end{array}$ & $\begin{array}{l}136 \\
124\end{array}$ & $\begin{array}{l}121 \\
103\end{array}$ & $\begin{array}{r}111 \\
95\end{array}$ & \\
\hline F. R. & 34 & $\stackrel{\mathrm{A}}{\mathrm{V}}$ & $\begin{array}{l}87 \\
85\end{array}$ & $\begin{array}{r}100 \\
96\end{array}$ & $\begin{array}{l}109 \\
105\end{array}$ & $\begin{array}{l}116 \\
111\end{array}$ & $\begin{array}{l}121 \\
116\end{array}$ & $\begin{array}{l}125 \\
121\end{array}$ & $\uparrow \begin{array}{l}144 \\
133\end{array}$ & $\begin{array}{l}157 \\
144\end{array}$ & $\begin{array}{l}168 \\
155\end{array}$ & $\begin{array}{l}177 \\
165\end{array}$ & $\begin{array}{l}167 \\
159\end{array}$ & $\begin{array}{l}159 \\
153\end{array}$ & $\begin{array}{l}153 \\
148\end{array}$ & $\begin{array}{l}148 \\
143\end{array}$ \\
\hline H. R. & 42 & $\begin{array}{l}\mathrm{A} \\
\mathrm{V}\end{array}$ & $\begin{array}{l}92 \\
88\end{array}$ & $\begin{array}{l}105 \\
101\end{array}$ & $\begin{array}{l}114 \\
112\end{array}$ & $\begin{array}{l}121 \\
119\end{array}$ & $\begin{array}{l}126 \\
125\end{array}$ & $\begin{array}{l}130 \\
129\end{array}$ & $\begin{array}{l}151 \\
144\end{array}$ & $\begin{array}{l}165 \\
159\end{array}$ & $\begin{array}{l}174 \\
170\end{array}$ & $\begin{array}{l}181 \\
179\end{array}$ & $\begin{array}{l}173 \\
170\end{array}$ & $\begin{array}{l}166 \\
162\end{array}$ & $\begin{array}{l}160 \\
156\end{array}$ & $\begin{array}{l}156 \\
153\end{array}$ \\
\hline A. S. & 22 & $\stackrel{\mathbf{A}}{\mathbf{V}}$ & $\begin{array}{l}86 \\
85\end{array}$ & $\begin{array}{r}100 \\
98\end{array}$ & $\begin{array}{l}108 \\
107\end{array}$ & $\begin{array}{l}114 \\
113\end{array}$ & $\begin{array}{l}118 \\
117\end{array}$ & $\begin{array}{l}122 \\
121\end{array}$ & $\begin{array}{l}142 \\
138\end{array}$ & $\begin{array}{l}156 \\
152\end{array}$ & $\begin{array}{l}167 \\
163\end{array}$ & $\begin{array}{l}176 \\
166\end{array}$ & $\begin{array}{l}165 \\
155\end{array}$ & $\begin{array}{l}154 \\
145\end{array}$ & $\begin{array}{l}144 \\
136\end{array}$ & $\begin{array}{l}134 \\
129\end{array}$ \\
\hline B. M. & 47 & $\stackrel{\mathbf{A}}{\mathrm{V}}$ & $\begin{array}{l}93 \\
89\end{array}$ & $\begin{array}{l}106 \\
103\end{array}$ & $\begin{array}{l}115 \\
111\end{array}$ & $\begin{array}{l}121 \\
117\end{array}$ & $\begin{array}{l}126 \\
121\end{array}$ & $\begin{array}{l}130 \\
124\end{array}$ & $\begin{array}{l}156 \\
140\end{array}$ & $\begin{array}{l}172 \\
154\end{array}$ & $\begin{array}{l}183 \\
162\end{array}$ & $\begin{array}{l}193 \\
170\end{array}$ & $\begin{array}{l}181 \\
162\end{array}$ & $\begin{array}{l}171 \\
151\end{array}$ & $\begin{array}{l}162 \\
141\end{array}$ & \\
\hline T. B. & 35 & $\stackrel{\mathrm{A}}{\mathrm{V}}$ & $\begin{array}{l}84 \\
81\end{array}$ & $\begin{array}{r}100 \\
95\end{array}$ & $\begin{array}{l}109 \\
104\end{array}$ & $\begin{array}{l}113 \\
107\end{array}$ & $\begin{array}{l}115 \\
107\end{array}$ & $\begin{array}{l}116 \\
108\end{array}$ & $\begin{array}{l}135 \\
122\end{array}$ & $\begin{array}{l}152 \\
137\end{array}$ & $\begin{array}{l}165 \\
149\end{array}$ & $\begin{array}{l}173 \\
160\end{array}$ & $\begin{array}{l}157 \\
143\end{array}$ & $\begin{array}{l}143 \\
127\end{array}$ & $\begin{array}{l}133 \\
114\end{array}$ & $\begin{array}{l}127 \\
108\end{array}$ \\
\hline C. $\mathrm{H}$. & 35 & $\stackrel{\mathrm{A}}{\mathbf{V}}$ & $\begin{array}{l}81 \\
81\end{array}$ & $\begin{array}{l}108 \\
104\end{array}$ & $\begin{array}{l}121 \\
116\end{array}$ & $\begin{array}{l}129 \\
125\end{array}$ & $\begin{array}{l}135 \\
131\end{array}$ & $\begin{array}{l}139 \\
135\end{array}$ & $\mid \begin{array}{l}169 \\
164\end{array}$ & $\begin{array}{l}186 \\
183\end{array}$ & $\begin{array}{l}200 \\
197\end{array}$ & $\begin{array}{l}212 \\
209\end{array}$ & $\begin{array}{l}202 \\
200\end{array}$ & $\begin{array}{l}194 \\
192\end{array}$ & $\begin{array}{l}187 \\
186\end{array}$ & $\begin{array}{l}181 \\
180\end{array}$ \\
\hline V.F. & 37 & $\stackrel{\mathrm{A}}{\mathrm{V}}$ & $\begin{array}{l}81 \\
78\end{array}$ & $\begin{array}{l}95 \\
90\end{array}$ & $\begin{array}{r}105 \\
98\end{array}$ & $\begin{array}{l}112 \\
102\end{array}$ & $\begin{array}{l}117 \\
106\end{array}$ & $\begin{array}{l}121 \\
108\end{array}$ & $\begin{array}{l}145 \\
118\end{array}$ & $\begin{array}{l}159 \\
140\end{array}$ & $\begin{array}{l}168 \\
153\end{array}$ & $\begin{array}{l}174 \\
161\end{array}$ & $\begin{array}{l}164 \\
150\end{array}$ & $\begin{array}{l}155 \\
141\end{array}$ & $\begin{array}{l}146 \\
135\end{array}$ & $\begin{array}{l}138 \\
133\end{array}$ \\
\hline E. A. & 27 & $\stackrel{\mathrm{A}}{\mathrm{V}}$ & $\begin{array}{l}84 \\
83\end{array}$ & $\begin{array}{l}98 \\
92\end{array}$ & $\begin{array}{r}109 \\
99\end{array}$ & $\begin{array}{l}116 \\
104\end{array}$ & $\begin{array}{l}121 \\
108\end{array}$ & $\begin{array}{l}124 \\
110\end{array}$ & $\begin{array}{l}143 \\
128\end{array}$ & $\begin{array}{l}154 \\
139\end{array}$ & $\begin{array}{l}164 \\
147\end{array}$ & $\begin{array}{l}172 \\
154\end{array}$ & $\begin{array}{l}150 \\
133\end{array}$ & $\begin{array}{l}135 \\
119\end{array}$ & $\begin{array}{l}127 \\
112\end{array}$ & $\begin{array}{l}122 \\
109\end{array}$ \\
\hline V. B. & 31 & $\stackrel{\mathrm{A}}{\mathrm{V}}$ & $\begin{array}{l}89 \\
87\end{array}$ & $\begin{array}{l}112 \\
107\end{array}$ & $\begin{array}{l}123 \\
118\end{array}$ & $\begin{array}{l}130 \\
126\end{array}$ & $\begin{array}{l}135 \\
131\end{array}$ & $\begin{array}{l}139 \\
134\end{array}$ & $\begin{array}{l}165 \\
157\end{array}$ & $\begin{array}{l}180 \\
171\end{array}$ & $\begin{array}{l}192 \\
183\end{array}$ & $\begin{array}{l}202 \\
193\end{array}$ & $\begin{array}{l}184 \\
178\end{array}$ & $\begin{array}{l}171 \\
167\end{array}$ & $\begin{array}{l}164 \\
161\end{array}$ & $\begin{array}{l}160 \\
157\end{array}$ \\
\hline
\end{tabular}

* Blood sugar values at 10-minute intervals taken from curves constructed from determinations on samples (30 to 40 per subject) drawn at staggered time intervals which varied in the different subjects

† A-Capillary blood sugar (mg. per cent), V-Antecubital vein sugar (mg. per cent).

$\uparrow$-Beginning of increased rate of glucose infusion. $\downarrow$-Resumption of initial speed of glucose infusion.

TABLE III

Miscellaneous control cases *

\begin{tabular}{|c|c|c|c|c|c|c|c|c|c|c|c|c|c|c|c|}
\hline \multirow[b]{2}{*}{ Patient } & \multirow[b]{2}{*}{ Age } & & \multicolumn{13}{|c|}{ Time in minutes } \\
\hline & & & 0 & 10 & 20 & 30 & 40 & 50 & 60 & 70 & 80 & 90 & 100 & 110 & 120 \\
\hline C. F. & 45 & $\begin{array}{l}\mathrm{A} t \\
\mathrm{~V} \dagger\end{array}$ & $\begin{array}{l}102 \\
100\end{array}$ & $\begin{array}{l}121 \\
118\end{array}$ & $\begin{array}{l}135 \\
132\end{array}$ & $\begin{array}{l}145 \\
142\end{array}$ & $\begin{array}{l}154 \\
151\end{array}$ & $\begin{array}{l}161 \\
158\end{array}$ & $\begin{array}{l}167 \\
164\end{array}$ & $\begin{array}{l}171 \\
168\end{array}$ & $\begin{array}{l}174 \\
170\end{array}$ & $\begin{array}{l}176 \\
172\end{array}$ & $\begin{array}{l}178 \\
173\end{array}$ & $\begin{array}{l}179 \\
174\end{array}$ & $\begin{array}{l}180 \\
175\end{array}$ \\
\hline P. H. & 30 & $\stackrel{\mathbf{A}}{\mathrm{V}}$ & $\begin{array}{l}95 \\
88\end{array}$ & $\begin{array}{l}112 \\
104\end{array}$ & $\begin{array}{l}124 \\
115\end{array}$ & $\begin{array}{l}133 \\
125\end{array}$ & $\begin{array}{l}140 \\
132\end{array}$ & $\begin{array}{l}146 \\
139\end{array}$ & $\begin{array}{l}150 \\
143\end{array}$ & $\begin{array}{l}154 \\
147\end{array}$ & $\begin{array}{l}156 \\
149\end{array}$ & $\begin{array}{l}159 \\
151\end{array}$ & $\begin{array}{l}162 \\
154\end{array}$ & $\begin{array}{l}164 \\
156\end{array}$ & \\
\hline G. M. & 22 & $\stackrel{A}{\mathrm{~A}}$ & $\begin{array}{l}86 \\
83\end{array}$ & $\begin{array}{l}110 \\
107\end{array}$ & $\begin{array}{l}128 \\
124\end{array}$ & $\begin{array}{l}141 \\
136\end{array}$ & $\begin{array}{l}150 \\
145\end{array}$ & $\begin{array}{l}157 \\
152\end{array}$ & $\begin{array}{l}161 \\
156\end{array}$ & & & & & & \\
\hline H. C. & 33 & $\begin{array}{l}\mathbf{A} \\
\mathbf{V}\end{array}$ & $\begin{array}{l}86 \\
83\end{array}$ & $\begin{array}{r}103 \\
98\end{array}$ & $\begin{array}{l}114 \\
109\end{array}$ & $\begin{array}{l}121 \\
116\end{array}$ & $\begin{array}{l}126 \\
121\end{array}$ & $\begin{array}{l}130 \\
124\end{array}$ & $\begin{array}{l}133 \\
127\end{array}$ & & & & & & \\
\hline D. Z. & 24 & $\begin{array}{l}\mathbf{A} \\
\mathbf{V}\end{array}$ & $\begin{array}{l}87 \\
85\end{array}$ & $\begin{array}{l}104 \\
100\end{array}$ & $\begin{array}{l}116 \\
110\end{array}$ & $\begin{array}{l}125 \\
117\end{array}$ & $\begin{array}{l}131 \\
123\end{array}$ & $\begin{array}{l}135 \\
127\end{array}$ & $\begin{array}{l}137 \\
131\end{array}$ & & & & & & \\
\hline R. G. & 36 & $\stackrel{\mathbf{A}}{\mathbf{V}}$ & $\begin{array}{l}87 \\
85\end{array}$ & $\begin{array}{l}109 \\
104\end{array}$ & $\begin{array}{l}121 \\
115\end{array}$ & $\begin{array}{l}127 \\
121\end{array}$ & $\begin{array}{l}131 \\
125\end{array}$ & $\begin{array}{l}133 \\
128\end{array}$ & & & & & & & \\
\hline R. B. & 29 & $\stackrel{\mathbf{A}}{\mathbf{V}}$ & $\begin{array}{l}97 \\
92\end{array}$ & $\begin{array}{l}109 \\
104\end{array}$ & $\begin{array}{l}120 \\
114\end{array}$ & $\begin{array}{l}129 \\
122\end{array}$ & $\begin{array}{l}134 \\
127\end{array}$ & $\begin{array}{l}138 \\
131\end{array}$ & & & & & & & \\
\hline C. J. & 26 & $\underset{\mathbf{V}}{\mathbf{A}}$ & $\begin{array}{l}87 \\
82\end{array}$ & $\begin{array}{r}102 \\
97\end{array}$ & $\begin{array}{l}116 \\
109\end{array}$ & $\begin{array}{l}126 \\
117\end{array}$ & $\begin{array}{l}134 \\
123\end{array}$ & $\begin{array}{l}139 \\
127\end{array}$ & & & & & & & \\
\hline
\end{tabular}

* These subjects received glucose infusion alone at one constant rate. Data treated as in Tables I and II.

† A-Capillary blood sugar (mg. per cent), V-Antecubital vein sugar (mg. per cent). 


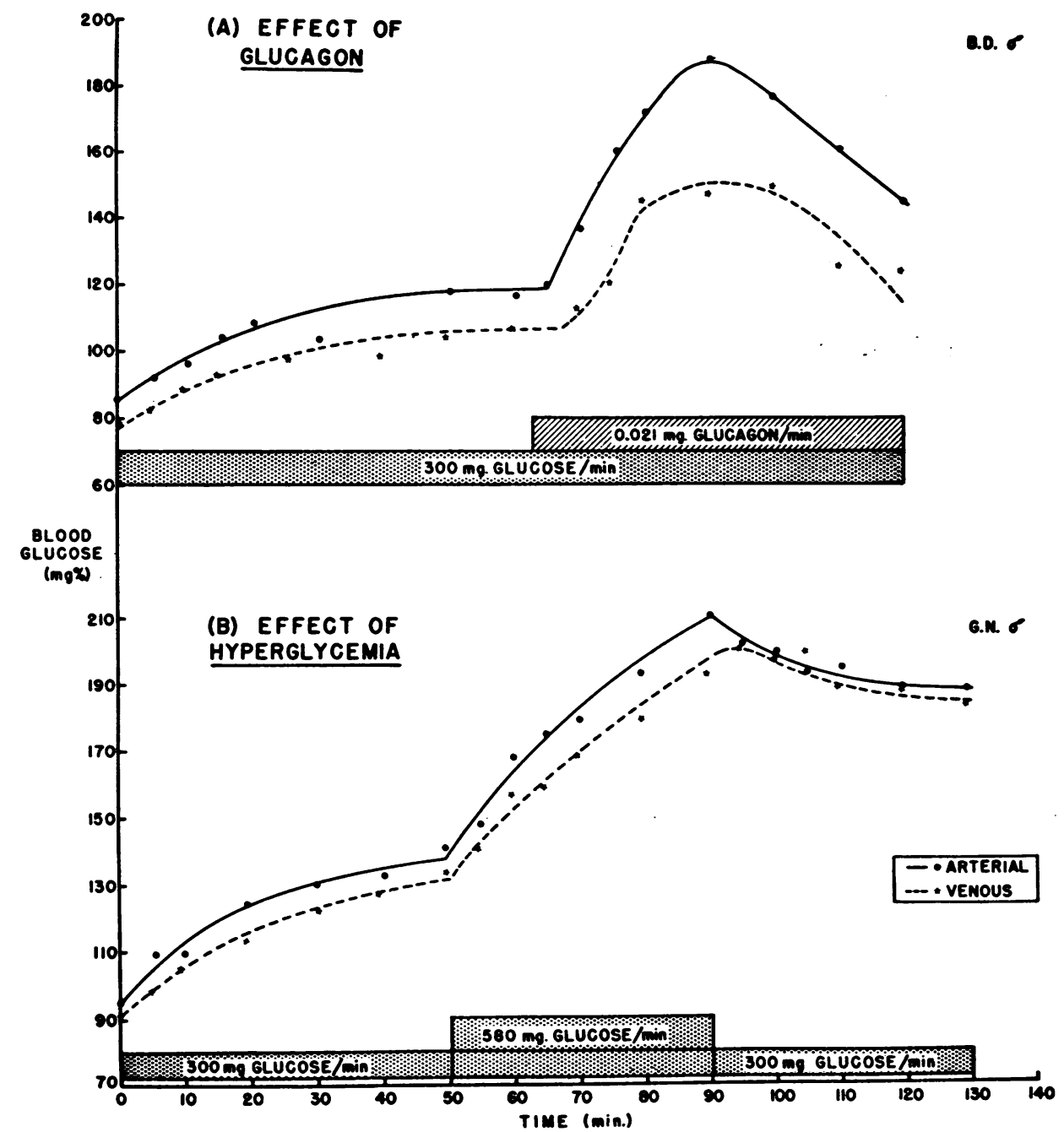

Fig. 1. Comparison between the Effects of (A) Glucagon and (B) Hyperglycemia Due to Glucose on Arterial and Venous Glucose Concentrations in Man

periods. The data were subjected to a median test employing a $2 \times 2$ contingency table (20). The resulting $\mathrm{chi}^{2}$ values showed that the increase in $A-V / A$ values during the glucagon periods was highly significant $(\mathrm{P}=.001)$. A similar statistical analysis was applied to the differences between the control and experimental periods for both the glucagon and glucose hyperglycemia cases. This revealed that glucagon caused a significantly greater $(P=.001)$ increase in $A-V / A$ than did glucose hyperglycemia. A comparison between the three groups of experimental periods is shown in Figure 2. These experiments demonstrate that the increased $\mathrm{A}-\mathrm{V}$ glucose differences observed with glucagon cannot be attributed to the increase in blood glucose levels per se. Further, it should be noted that the increased A-V/A values caused by glucagon persist throughout the test period despite the fall of blood sugar toward pre-glucagon levels in 11 of the 12 subjects.

Sodium space did not change following glucagon administration or when the rate of the glucose infusion was doubled. The mean value for the 20 subjects tested was $21.35 \pm 3.35$ per cent of body weight.

\section{Dog experiments}

Tables IV and V contain the data from the animal experiments. Glucagon causes a marked 


\section{THE EFFECT OF GLUCAGON ON THE PERIPHERAL UTILIZATION OF GLUCOSE}

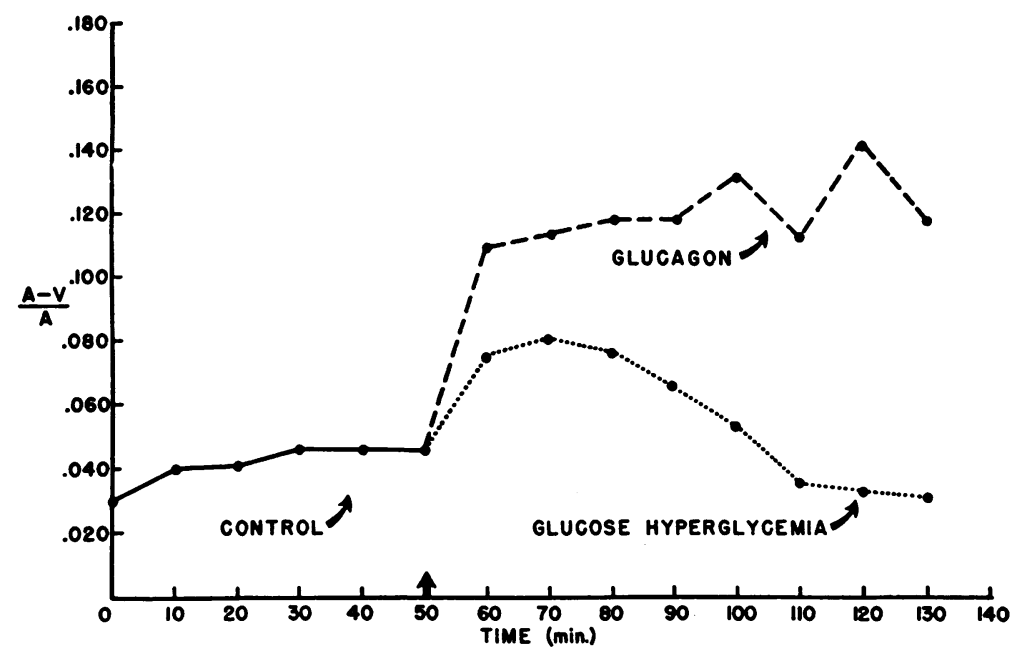

Fig. 2. A Summary of Continuous Infusion Experiments in Man Showing the Difference Between the Effect of Glucagon and of Glucose Hyperglycemia on the Peripheral Utilization of Glucose (A-V/A)

Each point of the control period represents the median value of 32 cases, whereas each point of the other two groups represents the median of 12 cases.

increase in $\mathrm{A}-\mathrm{V}$ glucose difference in the normal dog when given as a single injection or by continuous infusion (Figure 3 ). Similar results were observed in the depancreatized animal (Figure 4). As in the human, the increased $\mathrm{A}-\mathrm{V}$ glucose differences persist after the disappearance of the hyperglycemia.

\section{DISCUSSION}

Previous work from this laboratory, using a single glucose injection technique, suggested that glucagon causes a marked increase in A-V glucose differences in man (21). Recently, Van Itallie, Morgan, and Dotti (22) have reported experiments which support this finding. Both of these studies depended on the assumption that blood flow and $A-V$ glucose differences remain constant from day to day in a given individual. Actually, blood flow and A-V glucose differences may vary considerably in a given individual from day to day $(23,24)$. The present studies were designed to minimize these variables. Thus, both the control and hormone test periods were run in sequence within a $21 / 2$-hour period under stand- ardized basal conditions. Under these experimental conditions, the variations in $A-V / A$ values observed during the control periods were not significant. ${ }^{4}$ This is good evidence that blood flow was essentially constant. Glucagon itself (unlike adrenalin) has no cardiovascular effects $(1,2)$. Thus the A-V glucose differences observed in the present experiments may be considered proportional to glucose utilization in the forearm and hand.

The relationship of glucose utilization to the height of the arterial blood sugar is pertinent to this study, since glucagon causes a marked rise in arterial glucose concentration. The glucose infusion experiments indicate that peripheral utilization of glucose is proportional to arterial glucose levels in the range between 80 and $210 \mathrm{mg}$. per cent in agreement with previous work (16-18). Glucagon results in significantly greater glucose utilization at comparably elevated glucose levels.

4 A Rank Sign Test (25) of the significance of the variation in successive 10 -minute time periods revealed no significant variation beyond the first $10-\mathrm{min}$. interval. This appears obvious from Figure 2. 
TABLE IV

Effect of glucagon on blood sugar * of normal dogs

\begin{tabular}{|c|c|c|c|c|c|c|c|c|c|c|c|c|c|c|c|c|c|}
\hline \multirow{2}{*}{$\begin{array}{c}\text { Dog } \\
\text { number }\end{array}$} & & \multicolumn{16}{|c|}{ Time in minutes } \\
\hline & & $\mathbf{0}$ & 5 & 10 & 15 & 20 & 25 & 30 & 35 & 40 & 45 & 50 & 60 & 70 & 80 & 90 & 100 \\
\hline S† 564 & $\begin{array}{l}\text { A\| } \\
\text { V\| }\end{array}$ & $\begin{array}{l}81 \\
80\end{array}$ & $\begin{array}{l}82 \\
80\end{array}$ & $\begin{array}{l}82 \\
81\end{array}$ & $\begin{array}{l}84 \\
82\end{array}$ & $\begin{array}{l}116 \\
107\end{array}$ & $\begin{array}{l}146 \\
125\end{array}$ & $\begin{array}{l}160 \\
140\end{array}$ & $\begin{array}{l}167 \\
148\end{array}$ & $\begin{array}{l}159 \\
146\end{array}$ & $\begin{array}{l}146 \\
133\end{array}$ & $\begin{array}{l}134 \\
122\end{array}$ & $\begin{array}{l}112 \\
101\end{array}$ & $\begin{array}{l}97 \\
88\end{array}$ & $\begin{array}{l}92 \\
83\end{array}$ & & \\
\hline C 580 & A & $\begin{array}{l}87 \\
85\end{array}$ & $\begin{array}{l}88 \\
89\end{array}$ & $\begin{array}{l}89 \\
87\end{array}$ & & $\begin{array}{l}89 \\
88\end{array}$ & & $\begin{array}{r}102 \\
95\end{array}$ & & $\begin{array}{l}142 \\
120\end{array}$ & $\begin{array}{l}148 \\
138\end{array}$ & $\begin{array}{l}143 \\
132\end{array}$ & $\begin{array}{l}129 \\
116\end{array}$ & $\begin{array}{r}115 \\
99\end{array}$ & $\begin{array}{r}105 \\
82\end{array}$ & $\begin{array}{l}96 \\
79\end{array}$ & \\
\hline$S 435 b$ & $\stackrel{\mathbf{A}}{\mathbf{V}}$ & $\begin{array}{l}115 \\
103\end{array}$ & $\begin{array}{l}108 \\
103\end{array}$ & & $\begin{array}{l}109 \\
103\end{array}$ & $\begin{array}{l}146 \\
129\end{array}$ & $\begin{array}{l}171 \\
151\end{array}$ & & $\begin{array}{l}195 \\
172\end{array}$ & & $\begin{array}{l}188 \\
162\end{array}$ & & & & & & \\
\hline C 563 & $\begin{array}{l}\mathbf{A} \\
\mathbf{V}\end{array}$ & $\begin{array}{l}102 \\
101\end{array}$ & $\begin{array}{l}102 \\
100\end{array}$ & $\begin{array}{l}101 \\
100\end{array}$ & & $\begin{array}{l}101 \\
100\end{array}$ & & $\begin{array}{l}102 \\
100\end{array}$ & & $\begin{array}{l}146 \\
125\end{array}$ & & $\begin{array}{l}190 \\
165\end{array}$ & $\begin{array}{l}202 \\
164\end{array}$ & $\begin{array}{l}187 \\
156\end{array}$ & $\begin{array}{l}171 \\
148\end{array}$ & $\begin{array}{l}157 \\
138\end{array}$ & $\begin{array}{l}144 \\
128\end{array}$ \\
\hline S 529ł & $\begin{array}{l}\mathbf{A} \\
\mathbf{V}\end{array}$ & $\begin{array}{l}92 \\
85\end{array}$ & $\begin{array}{l}86 \\
83\end{array}$ & $\begin{array}{l}85 \\
85\end{array}$ & $\begin{array}{l}88 \\
86\end{array}$ & $\begin{array}{l}124 \\
102\end{array}$ & $\begin{array}{l}145 \\
124\end{array}$ & $\begin{array}{l}157 \\
132\end{array}$ & $\begin{array}{l}165 \\
139\end{array}$ & $\begin{array}{l}163 \\
139\end{array}$ & $\begin{array}{l}156 \\
137\end{array}$ & $\begin{array}{l}149 \\
134\end{array}$ & & & & & \\
\hline S 5298 & $\begin{array}{l}\mathbf{A} \\
\mathbf{V}\end{array}$ & $\begin{array}{l}88 \\
82\end{array}$ & $\begin{array}{l}91 \\
86\end{array}$ & $\begin{array}{l}88 \\
83\end{array}$ & $\begin{array}{l}89 \\
86\end{array}$ & $\begin{array}{r}115 \\
93\end{array}$ & $\begin{array}{l}125 \\
100\end{array}$ & $\begin{array}{l}128 \\
104\end{array}$ & $\begin{array}{l}126 \\
107\end{array}$ & $\begin{array}{l}123 \\
110\end{array}$ & $\begin{array}{l}120 \\
111\end{array}$ & $\begin{array}{l}115 \\
110\end{array}$ & & & & & \\
\hline
\end{tabular}

* Blood sugar values taken from curves which were constructed from values taken at staggered time intervals which varied in the different animals, and are expressed in $\mathrm{mg}$. per cent. $\uparrow$-Beginning of glucagon infusion.

$+\mathrm{S}$-Single injection of glucagon, $\mathrm{C}$-Continuous infusion of glucagon.

† 48-hr. fast.

86-hr. fast.

II A-Femoral artery, V-Femoral vein.

TABLE V

Effect of glucagon on blood sugar * of depancreatized dogs

\begin{tabular}{|c|c|c|c|c|c|c|c|c|c|c|c|c|c|c|c|c|c|c|}
\hline \multirow{2}{*}{\multicolumn{2}{|c|}{$\underset{\text { nog }}{\text { Dog }}$}} & & \multicolumn{16}{|c|}{ Time in minutes } \\
\hline & & & $\mathbf{0}$ & 5 & 10 & 15 & 20 & 25 & 30 & 35 & 40 & 45 & 50 & 60 & 70 & 80 & 90 & 100 \\
\hline St & $560 \ddagger$ & $\begin{array}{l}\mathrm{AT} \\
\mathrm{V}\end{array}$ & $\begin{array}{l}362 \\
356\end{array}$ & $\begin{array}{l}352 \\
356\end{array}$ & $\begin{array}{l}351 \\
351\end{array}$ & $\begin{array}{l}357 \\
353\end{array}$ & $\begin{array}{l}368 \\
357\end{array}$ & $\begin{array}{l}410 \\
386\end{array}$ & $\begin{array}{l}441 \\
413\end{array}$ & $\begin{array}{l}454 \\
430\end{array}$ & $\begin{array}{l}463 \\
440\end{array}$ & $\begin{array}{l}470 \\
447\end{array}$ & $\begin{array}{l}473 \\
453\end{array}$ & $\begin{array}{l}473 \\
454\end{array}$ & $\begin{array}{l}453 \\
432\end{array}$ & $\begin{array}{l}433 \\
411\end{array}$ & $\begin{array}{l}417 \\
396\end{array}$ & $\begin{array}{l}404 \\
384\end{array}$ \\
\hline $\mathbf{S}$ & 435ał & $\begin{array}{l}\mathbf{A} \\
\mathbf{V}\end{array}$ & $\begin{array}{l}270 \\
272\end{array}$ & $\begin{array}{l}272 \\
279\end{array}$ & $\begin{array}{l}274 \\
280\end{array}$ & $\begin{array}{l}275 \\
281\end{array}$ & $\begin{array}{l}277 \\
282\end{array}$ & $\begin{array}{l}316 \\
288\end{array}$ & $\begin{array}{l}353 \\
305\end{array}$ & $\begin{array}{l}379 \\
322\end{array}$ & $\begin{array}{l}394 \\
337\end{array}$ & $\begin{array}{l}404 \\
350\end{array}$ & $\begin{array}{l}410 \\
362\end{array}$ & $\begin{array}{l}418 \\
381\end{array}$ & $\begin{array}{l}419 \\
393\end{array}$ & $\begin{array}{l}413 \\
399\end{array}$ & & \\
\hline C & 435a‡ & $\begin{array}{l}\mathbf{A} \\
\mathbf{V}\end{array}$ & $\begin{array}{l}439 \\
423\end{array}$ & & $\begin{array}{l}431 \\
416\end{array}$ & & $\begin{array}{l}428 \\
410\end{array}$ & $\begin{array}{l}427 \\
412\end{array}$ & $\begin{array}{l}427 \\
415\end{array}$ & $\begin{array}{l}425 \\
423\end{array}$ & $\begin{array}{l}473 \\
431\end{array}$ & $\begin{array}{l}499 \\
450\end{array}$ & $\begin{array}{l}519 \\
473\end{array}$ & $\begin{array}{l}565 \\
506\end{array}$ & $\begin{array}{l}600 \\
526\end{array}$ & $\begin{array}{l}584 \\
529\end{array}$ & $\begin{array}{l}560 \\
525\end{array}$ & $\begin{array}{l}544 \\
518\end{array}$ \\
\hline $\mathbf{S}$ & $560 \ddagger$ & $\begin{array}{l}\mathbf{A} \\
\mathbf{V}\end{array}$ & $\begin{array}{l}310 \\
302\end{array}$ & & $\begin{array}{l}316 \\
306\end{array}$ & & $\begin{array}{l}322 \\
309\end{array}$ & & $\begin{array}{l}328 \\
311\end{array}$ & $\begin{array}{l}345 \\
314\end{array}$ & $\begin{array}{l}374 \\
338\end{array}$ & $\begin{array}{l}390 \\
355\end{array}$ & $\begin{array}{l}400 \\
365\end{array}$ & $\begin{array}{l}414 \\
383\end{array}$ & $\begin{array}{l}420 \\
390\end{array}$ & $\begin{array}{l}309 \\
393\end{array}$ & $\begin{array}{l}412 \\
392\end{array}$ & $\begin{array}{l}400 \\
388\end{array}$ \\
\hline C & $543 \ddagger$ & $\stackrel{\mathbf{A}}{\mathbf{V}}$ & $\begin{array}{l}235 \\
232\end{array}$ & $\begin{array}{l}230 \\
233\end{array}$ & $\begin{array}{l}233 \\
234\end{array}$ & $\begin{array}{l}234 \\
234\end{array}$ & $\begin{array}{l}230 \\
230\end{array}$ & & $\begin{array}{l}286 \\
252\end{array}$ & $\begin{array}{l}310 \\
283\end{array}$ & $\begin{array}{l}323 \\
304\end{array}$ & $\begin{array}{l}332 \\
315\end{array}$ & $\begin{array}{l}337 \\
324\end{array}$ & $\begin{array}{l}334 \\
324\end{array}$ & $\begin{array}{l}329 \\
322\end{array}$ & $\begin{array}{l}323 \\
317\end{array}$ & $\begin{array}{l}316 \\
310\end{array}$ & $\begin{array}{l}309 \\
303\end{array}$ \\
\hline C & 5608 & $\stackrel{\mathbf{A}}{\mathbf{V}}$ & $\begin{array}{l}75 \\
72\end{array}$ & & $\begin{array}{l}79 \\
74\end{array}$ & & $\begin{array}{l}82 \\
76\end{array}$ & $\begin{array}{l}85 \\
82\end{array}$ & $\begin{array}{r}128 \\
96\end{array}$ & $\begin{array}{l}175 \\
129\end{array}$ & $\begin{array}{l}199 \\
157\end{array}$ & $\begin{array}{l}215 \\
174\end{array}$ & $\begin{array}{l}226 \\
185\end{array}$ & $\begin{array}{l}239 \\
203\end{array}$ & $\begin{array}{l}248 \\
215\end{array}$ & $\begin{array}{l}253 \\
225\end{array}$ & $\begin{array}{l}257 \\
231\end{array}$ & $\begin{array}{l}260 \\
234\end{array}$ \\
\hline $\mathbf{S}$ & 435a\| & $\stackrel{\mathbf{A}}{\mathbf{V}}$ & $\begin{array}{l}62 \\
60\end{array}$ & $\begin{array}{l}60 \\
58\end{array}$ & $\begin{array}{l}56 \\
53\end{array}$ & $\begin{array}{l}58 \\
51\end{array}$ & $\begin{array}{l}60 \\
48\end{array}$ & $\begin{array}{r}128 \\
95\end{array}$ & $\begin{array}{l}200 \\
147\end{array}$ & $\begin{array}{l}255 \\
190\end{array}$ & $\begin{array}{l}280 \\
217\end{array}$ & $\begin{array}{l}300 \\
229\end{array}$ & $\begin{array}{l}317 \\
241\end{array}$ & $\begin{array}{l}338 \\
257\end{array}$ & & & & \\
\hline $\mathbf{S}$ & 435a\| & $\stackrel{\mathbf{A}}{\mathbf{V}}$ & $\begin{array}{l}87 \\
80\end{array}$ & $\begin{array}{l}90 \\
72\end{array}$ & $\begin{array}{l}89 \\
78\end{array}$ & $\begin{array}{l}89 \\
84\end{array}$ & $\begin{array}{r}119 \\
99\end{array}$ & $\begin{array}{l}139 \\
112\end{array}$ & $\begin{array}{l}152 \\
124\end{array}$ & $\begin{array}{l}162 \\
131\end{array}$ & $\begin{array}{l}168 \\
142\end{array}$ & $\begin{array}{l}173 \\
148\end{array}$ & $\begin{array}{l}178 \\
152\end{array}$ & $\begin{array}{l}184 \\
157\end{array}$ & & & & \\
\hline $\mathbf{S}$ & 435a\| & $\begin{array}{l}\mathbf{A} \\
\mathbf{V}\end{array}$ & $\begin{array}{l}69 \\
69\end{array}$ & $\begin{array}{l}78 \\
72\end{array}$ & $\begin{array}{l}89 \\
75\end{array}$ & $\begin{array}{l}73 \\
68\end{array}$ & $\begin{array}{r}114 \\
94\end{array}$ & $\begin{array}{l}143 \\
113\end{array}$ & $\begin{array}{l}158 \\
127\end{array}$ & $\begin{array}{l}167 \\
136\end{array}$ & $\begin{array}{l}177 \\
143\end{array}$ & $\begin{array}{l}184 \\
149\end{array}$ & $\begin{array}{l}189 \\
153\end{array}$ & $\begin{array}{l}194 \\
157\end{array}$ & & & & \\
\hline
\end{tabular}

* Blood sugar values taken from curves which were constructed from values taken at staggered time intervals which varied in the different animals and are expressed in $\mathrm{mg}$. per cent. $\uparrow$-Beginning of glucagon infusion.

$\dagger \mathrm{S}-$ Single injection of glucagon, $\mathrm{C}-$ Continuous infusion of glucagon.

$\ddagger$ Experiment started 16 hours after last food and insulin (regular).

Experiment started 11 hours after last food and insulin (regular).

Experiment started 4 hours after last food and insulin (regular).

A-Femoral artery, V-Femoral vein. 
THE EFFECT OF GLUCAGON IN THE NORMAL DOG

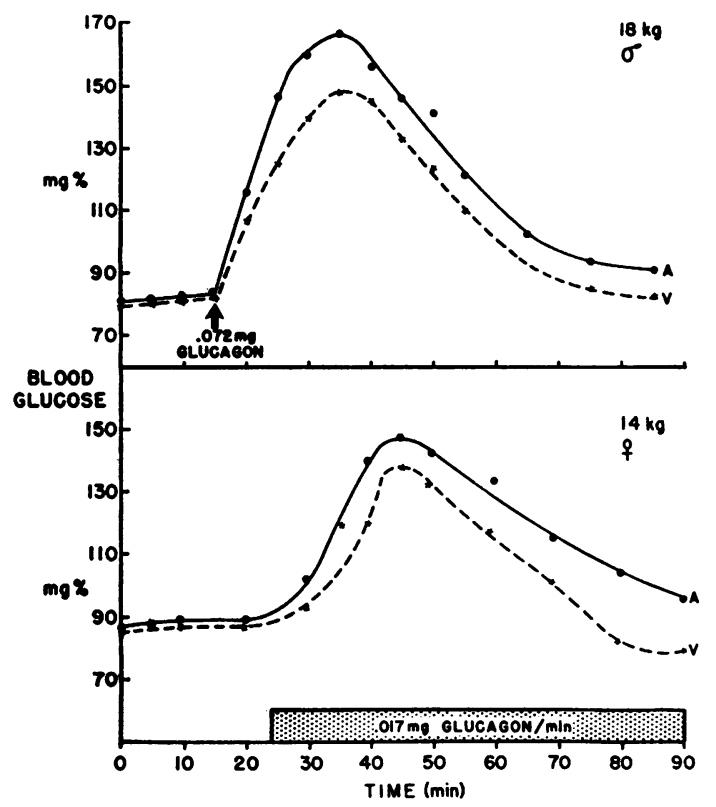

Fig. 3. The Effect of a Single I.V. Injection of Glucagon and of Continuous I.V. Glucagon Infusion (in Normal Saline) on (Femoral) Arterial and Venous Glucose Levels in the Normal Dog
These observations are of considerable importance from the standpoint of glucagon's potential role in the regulation of carbohydrate metabolism. They indicate that glucagon has two integrated actions; namely, the mobilization of liver glycogen and the enhancement of the peripheral utilization of glucose. The persistence of the peripheral effect beyond the disappearance of the hyperglycemia suggests that glucagon has a more prolonged action than hitherto believed. The occurrence of these two effects of glucagon in preliminary experiments in the depancreatized dog indicates that the effects are independent of insulin action.

There is some experimental evidence which suggests that pituitary growth hormone causes the release of glucagon and that part of the diabetogenic action of the former is due to the released glucagon $(1,2)$. This hypothesis is not supported by the present experiments which show that glucagon increases the peripheral utilization of glucose; whereas there is abundant evidence that growth hormone decreases carbohydrate utilization (26).

The lack of change in $\mathrm{Na}^{22}$ space with glucagon

\section{THE EFFECT OF GLUCAGON IN}

\section{A DEPANCREATIZED DOG}

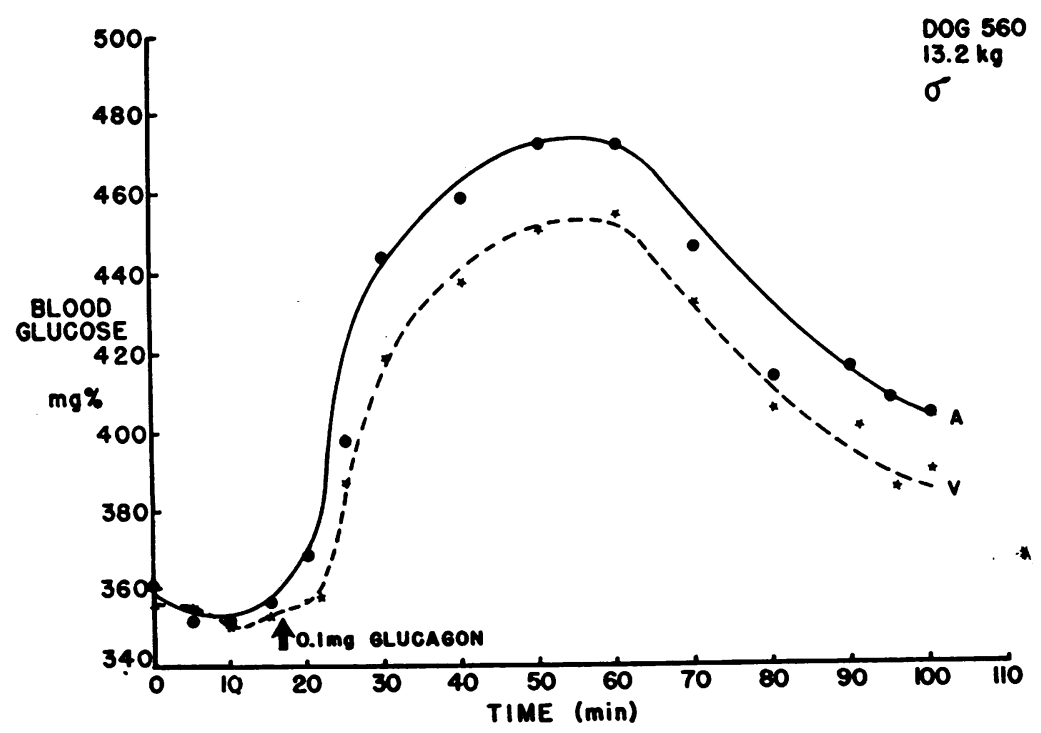

Fig. 4. The Effect of a Single I.V. Injection of Glucacon on (Femoral) Arterial and Venous Glucose Levels in the Depancreatized DoG

Experiment was done 16 hours after the last dose of insulin and food. 
or glucose infusions at two different infusion rates indicates that no significant alteration in extracellular space has taken place during the experiments.

\section{SUMMARY AND CONCLUSIONS}

Studies on the effect of glucagon on blood glucose utilization in normal men using a constant glucose infusion technique have been presented. They show that glucagon causes a highly significant increase in the peripheral utilization of glucose which persists after the disappearance of the hyperglycemia. This increased glucose utilization is significantly greater than that observed with the hyperglycemia produced by glucose alone. The findings suggest that glucagon may fulfil a dual role in carbohydrate metabolism by producing hyperglycemia through mobilization of liver glycogen and concomitantly increasing the peripheral utilization of blood glucose. They also indicate that glucagon has a more prolonged action than previously thought.

Preliminary studies in normal and depancreatized dogs are also reported. Both groups of animals respond to glucagon in the same way as the normal human subject. These experiments indicate that the enhancing action of glucagon on the peripheral utilization of glucose does not depend upon a release of insulin.

\section{APPENDIX}

In the normal dog (18) it has been shown that the peripheral utilization of glucose $(P)$ is a linear function of arterial glucose concentration (A). Therefore:

or

$$
P \propto A
$$

$$
P=k A \text {, }
$$

where $\mathrm{k}$ is the proportionality constant. At any time $\mathrm{t}$ :

$$
P=R(A-V),
$$

where $R$ is the blood flow and $V$ is the venous blood glucose concentration. If (1) and (2) are equated, it may be shown that:

$$
\frac{A-V}{A}=K \text { (constant), }
$$

where $\mathrm{K}$ is a function of the specific rate constant of peripheral glucose utilization, the volume of glucose distribution and peripheral blood flow. If blood flow and glucose distribution remain constant, then $A-V / A$ must remain constant unless the rate constant of peripheral glucose utilization changes.

\section{ACKNOWLEDGMENTS}

We are indebted to Dr. C. Mackenzie for advice in the preparation of the manuscript, to Drs. L. Bernstein and W. Crow for assistance in the statistical analyses of the data, to Dr. F. M. Rachiele for performing the pancreatectomies, and to F. Stoll for assistance in the animal experiments.

\section{REFERENCES}

1. DeDuve, C., Glucagon, the hyperglycæmic glycogenolytic factor of the pancreas. Lancet, 1953, 2, 99.

2. Foà, P. P., Glucagon, the hyperglycemic glycogenolytic hormone of the pancreas. Advances Int. Med. 1954, 6, 29.

3. Staub, A., Sinn, L., and Behrens, O. K., Purification and crystallization of hyperglycemic glycogenolytic factor (HGF). Science, 1953, 117, 628.

4. Staub, A., Sinn, L., and Behrens, O. K., Purification and crystallization of glucagon. J. Biol. Chem., 1955, 214, 619.

5. Sutherland, E. W., and Cori, C. F., Effect of hyperglycemic glycogenolytic factor and epinephrine on liver phosphorylase. J. Biol. Chem., 1951, 188, 531.

6. Sutherland, E. W., Wosilait, W. D., and Rall, T., Effect of epinephrine and glucagon on activation of liver phosphorylase. Federation Proc., 1955, 14, 289.

7. Drury, D. R., Wick, A. N., and Sherrill, J. W., The effect of the hyperglycemic factor on the metabolism of glucose by the extrahepatic tissues. Diabetes, 1954, 3, 129.

8. Bürger, M., Insulin und glukagon. Ztschr. f. d. ges. innere Med. u. Grenzgeberta, 1947, 2, 311.

9. Pincus, I. J., and Rutman, J. Z., Glucagon, the hyperglycemic agent in pancreatic extracts; a possible factor in certain types of diabetes. Arch. Int. Med., 1953, 92, 666.

10. Waters, E. T., Review of studies on blood sugar. Bull. New York Acad. Med., 1949, 25, 32.

11. Conn, J. W., Endocrine regulation of the blood sugar. Ann. Int. Med., 1953, 38, 179.

12. Nelson, N., A photometric adaptation of the Somogyi method for the determination of glucose. J. Biol. Chem., 1944, 153, 375.

13. Goss, C. M., Ed., Gray's Anatomy of the Human Body. 26th ed., Philadelphia, Lea \& Febiger, 1954, p. 737.

14. Schaeffer, J. P., Ed., Morris' Human Anatomy; A Complete Systematic Treatise. 11th ed., New York, Blakiston, 1953, p. 774.

15. Woerdeman, M. W., Atlas of Human Anatomy, Descriptive and Regional. New York, Blakiston, 1950, Vol. II, p. 346.

16. Somogyi, M., Studies of arteriovenous differences in blood sugar. I. Effect of alimentary hyperglycemia 
on the rate of extrahepatic glucose assimilation. J. Biol. Chem., 1948, 174, 189.

17. Soskin, S., and Levine, R., A relationship between the blood sugar level and the rate of sugar utilization, affecting the theories of diabetes. Am. J. Physiol., 1937, 120, 761.

18. Lang, S., Goldstein, M. S., and Levine, R., Influence of the liver on uptake of glucose by extrahepatic tissues. Am. J. Physiol., 1954, 177, 447.

19. Hlad, C. J., Elrick, H., Huffman, E., and Whipple, N., Studies on $\mathrm{Na}^{22}$ space in man. In preparation.

20. Mood, A. M., Introduction to the Theory of Statistics. New York, McGraw-Hill Book Co., Inc., 1950, Chap. 16, pp. 385-418.

21. Elrick, H., Discussion on influence of growth hormone on the mammary gland and on human metabolism in International Symposium, The Hypophyseal
Growth Hormone, Nature and Actions, New York, Blakiston, 1955, pp. 561-568.

22. Van Itallie, T. B., Morgan, M. C., and Dotti, L. B., Effect of glucagon on peripheral utilization of glucose in man. J. Clin. Endocrinol. \& Metab., 1955, $15,28$.

23. Elrick, H., Hlad, C. J., Smith, A., and Bow, T., Unpublished observations.

24. Cooper, K. E., Edholm, O. G., and Mottram, R. F., The blood flow in skin and muscle of the human forearm. J. Physiol., 1955, 128, 258.

25. Wilcoxon, F., Some Rapid Approximate Statistical Procedures. New York, Am. Cyanamid Co., 1949, $16 \mathrm{p}$.

26. deBodo, R. C., and Sinkoff, M. W., The role of growth hormone in carbohydrate metabolism. Ann. New York Acad. Sc., 1953, 57, 23. 